\title{
Geometric illusions as a function of pigmentation of the Fundus oculi and target size*
}

\author{
CHRISTOPHER A. BAYER $\dagger$ and A. W. PRESSEY \\ University of Manitoba, Winnipeg, Canada
}

A within-race design was employed in which two forms of the Mueller-Lyer illusion and the vertical-horizontal illusion were studied as a function of pigmentation density of the eye and target size. It was found that the illusions did not vary as a function of pigmentation density. In addition, with an increase in target size the ingoing Mueller-Lyer and vertical-horizontal illusions remained relatively constant but the outgoing Mueller-Lyer illusion decreased. Explanations are offered for both sets of results.

In 1967 , Pollack and Silvar measured the outgoing form of the Mueller-Lyer illusion in children ( 8 to 14 years of age) who differed in pigmentation of the Fundus oculi. They concluded that degree of pigmentation was highly related to magnitude of illusion; i.e., deeply pigmented Os exhibited a smaller illusion than did lightly pigmented Os. However, scepticism about such a conclusion is generated by the kind of experiment they conducted. For one thing, Pollack and Silvar employed what is, in essence, a between-race design. Eighty-seven percent of the Os in the darkly pigmented group were Negro, and $95 \%$ of the lightly pigmented group were Caucasian. It is possible that racial and cultural variables other than pigmentation produced the differences that they obtained. A second weakness in their experiment is that they failed to provide measurements of a control line in the different pigmentation groups. A control condition is especially important when organismic variables are being investigated. For example, it is possible that pigmentation affects measurements of nonillusory targets. If lines alone are overestimated by lightly pigmented Os, then the results obtained by Pollack and Silvar would be explained without any reference to illusions. The major purpose of this experiment was to measure geometric illusions only in Caucasian Os who varied in degree of pigmentation and to provide control conditions to assess whether measurements of nonillusory targets vary as a function of pigmentation.

* This study is based on a Master's thesis submitted by C. A. Bayer to the University of Manitoba. It was supported in part by a National Reserach Council of Canada grant (APA-177) to the second author.

+Requests for reprints should be sent to Christopher A. Bayer, Department of Psychology, University of Manitoba, Winnipeg 19 , Canada.
A second objective was to assess the generality of Pollack and Silvar's findings. Specifically, we sought to determine whether pigmentation affects different types of illusions and whether pigmentation interacts with a stimulus variable which is known to have a strong effect on magnitude of illusion-viz, target size. The types of illusions chosen were (1) the outgoing Mueller-Lyer, (2) the ingoing Mueller-Lyer, and (3) the vertical-horizontal illusions.

\section{SUBJECTS}

Seventy-four men enrolled in introductory psychology at the University of Manitoba served as Os. The sample was restricted to Caucasian Manitobans between the ages of 18 and 22 years who had normal vision with or without glasses. The mean age was 20.4 years, and Os who normally wore glasses did so during testing.

\section{APPARATUS}

The apparatus consisted of a white target holder, $36.8 \mathrm{~cm}$ wide and $39.5 \mathrm{~cm}$ high. The face of the holder was tilted backward $20 \mathrm{deg}$ from the perpendicular. A chinrest was placed in front of the holder (and perpendicular to the line of sight) so that the distance between O's eyes and the face of the frame was about $41 \mathrm{~cm}$. One lamp (lit by a $100-\mathrm{W}$ blue light bulb) was placed on each side of the target holder about $25 \mathrm{~cm}$ away from, but perpendicular to, the side of the holder.

Nine illusory targets and three control targets were prepared with black ink and printed separately on $21.5 \times 28.0 \mathrm{~cm}$ sheets of white paper. Three ingoing and three outgoing forms of the Mueller-Lyer illusion, in vertical orientation, were used. The distance between apices for each form was 10,35 , and $60 \mathrm{~mm}$. The obliques were one-quarter of the length between apices and the interior angles formed by the obliques were $90 \mathrm{deg}$. Each target was centered on an imaginary point $60 \mathrm{~mm}$ from the top and $14 \mathrm{~cm}$ from either side of the sheet. A dot was located directly to the right and slightly below the top apex of each target for the purpose of orienting O's response, since a method of production was employed. For example, in the $10-\mathrm{mm}$ target the dot was $10 \mathrm{~mm}$ to the right and $2.5 \mathrm{~mm}$ below the top apex. As target size increased, the dot was moved so that it was in the same relative location for each target.

Three targets of the vertial-horizontal illusion were used. The lengths of the horizontal components (which were drawn parallel to the top of the sheet) were 10,35 , and $60 \mathrm{~mm}$. A dot was drawn in the middle of each horizontal line, and this dot corresponded to the imaginary center employed in the Mueller-Lyer targets. The three control targets were identical to the Mueller-Lyer targets, except that the obliques were replaced by dots. The distances between dots were 10,35 , and $60 \mathrm{~mm}$.

PROCEDURE

The Os were divided into five groups on the basis of relative perceived darkness (density) of the pigmentation of the Fundus oculi. The area of the retina viewed was between the macula lutea and the optic disk. This area was chosen for two reasons: (1) the relative absence of blood vessels on the retinal surface, and (2) the ease with which this area can be located consistently and focused upon. An optometrist, 1 using a battery-handle May ophthalmoscope, performed the ratings in a dark room. The categorization of Os was judgmental. Initially, Os were distinguished on a 3-point basis: "light," "medium," and "dark." As the number of Os examined increased, "light-medium" and "medium-dark" categories were also able to be included. There were $15,9,21,15$, and 14 Os in Groups 1 (light) to 5 (dark), respectively.

The Os sat in front of the apparatus and were shown the $10-\mathrm{mm}$ version of each target. For the Mueller-Lyer targets, it was explained that the task was to mark a point directly below the dot so that the distance he produced appeared equal to the distance between apices. For the horizontal line, he was asked to draw a dot directly below the bisecting dot so that the distance between dots appeared equal to the horizontal line. For the control cards, the same response was required, except that the distance between dots was to be reproduced. It was emphasized that only one response could be made on each target and that judgments were to be made visually, i.e., arm movements to aid the judgment of length were not 
allowed. Moreover, responses were to be made only after a signal was given.

Each $\mathrm{O}$ received all combinations of type and size of target. The stimuli were presented in four consecutive blocks of 12 targets in each block. The order of targets was randomized within blocks, between blocks, and between Os.

A memory drum, set at $5 \mathrm{sec}$, controlled viewing time. It was started as soon as the target was placed on the stand, and a loud click signaled $O$ to make his response. The sheet was then removed and a new trial began when the next target was placed on the stand. The illuminance of the stimuli, at the face of the holder, was $10 \mathrm{fc}$. RESULTS

Reproductions of length were measured by a transparent millimeter scale, and these measurements were accurate to within $0.5 \mathrm{~mm}$. In the first analysis, the control scores were subjected to an analysis of variance. Only target size had a significant effect. Pigmentation had no effect on the control scores and, even when Groups 1 and 2 were compared with Groups 4 and 5 , no discernible trends were present.

Magnitude of illusion was calculated by subtracting the mean control score from the mean illusion score for each of the nine targets. These scores were then divided by the standard distance to obtain a "percent illusion." An analysis of variance, in which the main effects were type of illusion, target size, and fundus density, was carried out. The $F$ value for type of illusion was $41.96(\mathrm{df}=2 / 138 ; \mathrm{p}<.01)$ and for target size it was $5.30(\mathrm{df}=2 / 138$; $\mathrm{p}<.01$ ). The $\mathrm{F}$ value for the Type of nlusion by Size interaction was 3.57 $(\mathrm{df}=4 / 276 ; \mathrm{p}<.05)$. None of the remaining $F$ values was significant at the .05 level.

In a second analysis, the scores for the vertical-horizontal illusion were removed and an analysis of variance was again performed. The $F$ value for target size was 9.83 ( $\mathrm{df}=2 / 138$; $\mathrm{p}<.01$ ) and for Type of Illusion by Size interaction it was 6.11 $(\mathrm{df}=2 / 138 ; \mathrm{p}<.01)$. None of the remaining $F$ values was significant at the .05 level.

It is clear from these analyses that fundus density had no effect on the magnitude of either of the Mueller-Lyer illusions or the vertical-horizontal illusion. Once again, the extreme fundus density groups were blocked, and, as with the control scores, no systematic trends were evident.

The relationship of illusion to target size is shown in Fig. 1. The outstanding feature of these results is that the ingoing Mueller-Lyer and the vertical-horizontal illusions do not

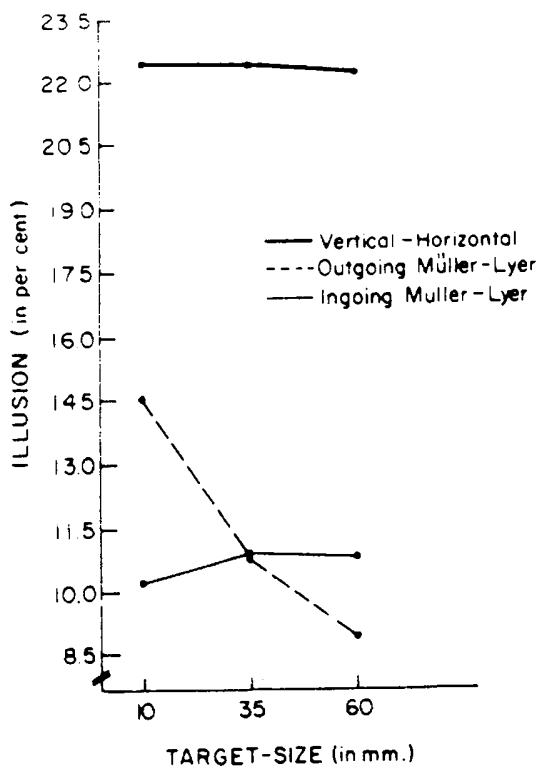

Fig. 1. The vertical-horizontal and Mueller-Lyer illusions as a function of target size.

change as target size increases, but the outgoing Mueller-Lyer illusion decreases.

\section{DISCUSSION}

The results of this study do not support the conclusion by Pollack and Silvar that fundus density affects the magnitude of geometric illusions. This difference could be due to the fact that, in Pollack and Silvar's study, the critical variable was not pigmentation of the Fundus oculi but some other racial or cultural variable. It could also be argued that, within a Caucasian group, pigmentation does not vary enough to produce significant differences in illusion. However, if this were so, it would be extremely difficult to test the relationship between pigmentation and illusion without confounding other variables. Indeed, the strategy of blocking Os on the basis of rated pigmentation would not be an appropriate one.

One might also argue that the difference between the two studies is due to the different populations employed. However, conversion of Pollack and Silvar's data (i.e., the mean magnitude of illusion) into percent illusion form indicates that lightly pigmented children yielded a $17.68 \%$ illusion on the outgoing Mueller-Lyer form, while darkly pigmented children yielded a $12.86 \%$ illusion on the same form. In the present study, Caucasian adults yielded a $14.50 \%$ illusion on the outgoing Mueller-Lyer form of approximately the same visual angle (i.e., the $10-\mathrm{mm}$ form) as that used by Pollack and Silvar. The Mueller-Lyer illusion is a Piaget (1969) Type I illusion--it decreases with age. This finding is based on data mainly from Caucasian populations. Since Pollack and Silvar's lightly pigmented group was $95 \%$ Caucasian, the expected decrease in illusion (from $17.68 \%$ to $14.50 \%$ ) with age is demonstrated when the previous study's data is compared to the data presented here. Based on this comparison, it seems reasonable to assume that the data of the present study is reliable.

Finally, it could be argued that the difference between the two studies is due to the different psychophysical methods employed. It is not immediately obvious why pigmentation should interact with psychophysical method, but even if it were true, the results of this study would indicate that pigmentation plays a role only under very limited conditions and, as such, is probably not an important variable in the perception of illusions.

The results on the effects of target size are consistent with previous findings, but they also throw new light on the matter. Begelman \& Steinfeld (1967) investigated the effect of target size on the vertical-horizontal illusion and concluded that the illusion decreases as a function of size if the horizontal portion is the variable stimulus but remains relatively constant if the vertical line is variable. Since in this study judgments were made (of a horizontal standard) along a vertical dimension, the results are consistent with those obtained by Begelman and Steinfeld.

Two studies have been carried out in which target size was varied in the Mueller-Lyer illusion (Binet, 1895; Heymans, 1896). Both of these investigators used a method of measuring the ingoing illusion with the outgoing illusion, or vice versa, and both concluded that the Mueller-Lyer illusion decreased with an increase in target size. The results of the present study show that it is the outgoing illusion which produces the decrease.

The assimilation theory of geometric illusions (Pressey, 1971) provides a relatively simple explanation of the interaction between forms of the Mueller-Lyer illusion and target size. This theory states that the Mueller-Lyer illusion is due to the assimilation of the standard line to either the shorter or longer magnitudes enclosed by the obliques. Moreover, it states that the effectiveness of a context decreases as the distance from the center of the field increases (Pressey, Butchard, \& Scrivner, in press). Operationally, the center of a field is defined by determining the most extreme elements to be judged (as set by instructions) and calculating the midpoint of these elements. 
Now, when a very small outgoing Mueller-Lyer illusion is employed, the obliques are located near the center of the field and will contribute a great deal to the judgment of the standard distance. However, as target size increases, the tips of the obliques move further and further from the center of the field and hence become fairly ineffective in altering the percept. In other words, in a very large target, it becomes relatively easy to "gate" out the context by the simple procedure of not directing eye movements to the obliques. It should be noted that, according to this analysis, increasing target size has roughly the same effect as decreasing the length of the outgoing obliques, and therefore, the illusion should decline with an increase in target size.

The ingoing Mueller-Lyer illusion, on the other hand, should not change as a function of target size. The ingoing obliques are always oriented towards the center of the field. No matter how large the target, if the $O$ is to judge its length he must process information from one extreme edge to the other extreme edge of the standard distance. As he does so, the ingoing obliques fall in the same relative position to the center of the field and hence remain of constant effectiveness.

The final point worth mentioning about the data is that, in the very large targets, the ingoing illusion is larger than the outgoing illusion. Many investigators have reported that it is the outgoing illusion which produces a larger effect. As argued in an earlier paper (Butchard \& Pressey, 1971), the proper conclusion appears to be that the form of illusion which is larger depends upon stimulus variables such as length of obliques, angle of obliques, ${ }^{2}$ and target size.

\section{REFERENCES}

BEGELMAN D \& STEINFELD, G. An investigation of several parameters of the horizontal-vertical illusion. Perception \& Psychophysics, 1967, 2, 539-543.

BINET, A. La mèsures des illusions visuèlles chez les enfants. Revue Philosophique, $1895,40,11-25$.
BUTCHARD, N., \& PRESSEY,A. W. The effects of closing lines on the two forms of the Muller-Lyer illusion. Psychonomic Science, 1971, 23, 257-258.

HEYMANS, G. Quantitaetive Untersuchungen ueber das "optische Paradoxon." Zeitschrift fur Psychologie und Physiologie der Sinnesorgane, 1896. 9,221-255

PIAGET, J. The mechanisms of perception. Trans. G. N. Seagrim. London: Routledge \& Kegan Paul, 1969.

POLLACK, $\mathrm{R}, \mathrm{H}$ \& SILVAR, S. Magnitude of the Mueller-Lyer illusion in children as a function of pigmentation of the Fundus oculi. Psychonomic Science, 1967, 8. 83-84.

PRESSEY, A. W. An extension of assimilation theory to illusions of size, area, and direction. Perception \& Psychophysics, 1971,9,172-176.

PRESSEY, A. W., BUTCHARD, N., \& SCRIVNER, L. Assimilation theory and the Ponzo illusion: Quantitative predictions. Canadian Joumal of Psychology, in press. NOTES

1. We are indebted to David Bryer, O.D. and E. M. Finkleman, O.D., for their assistance. Dr. Bryer performed the fundus ratings. We are also indebted to Drs. F. Shively and L. Shewchuk for their critical evaluation of the thesis.

2. Unpublished data by C. A. Bayer and

A W. Pressey entitled "Analysis of two forms of the Müller-Lyer illusion." 